\title{
Pressurized fluid flow within the mechanical stability domain of fault zones in shale
}

\author{
Frédéric-Victor Donzé ${ }^{1, *}$, Alexandra Tsopela ${ }^{1}$, Yves Guglielmi ${ }^{2}$, Pierre Henry ${ }^{3}$, and Claude \\ Gout $^{4}$ \\ ${ }^{1}$ Université Grenoble Alpes, Université. Savoie Mont Blanc, CNRS, IRD, IFSTTAR, ISTerre, 38000 \\ Grenoble, France \\ ${ }^{2}$ Lawrence Berkeley National Laboratory, Earth and Environmental Science Area, 1 Cyclotron road, \\ M/S 74R316C, Berkeley, CA 94720 \\ ${ }^{3}$ CEREGE, Aix-Marseille University-CNRS-IRD, Marseille, France \\ ${ }^{4}$ Total S.A., Pau, France
}

\begin{abstract}
Fracture interaction mechanisms and reactivation of natural discontinuities under fluid pressurization conditions inside fault zone can represent critical issues in risk assessment of caprock integrity. A field injection test, carried out in a damage fault zone at the decameter scale i.e. mesoscale, has been studied using a Distinct Element Model. Considering the complex structural nature of a fault zone, the contribution of fracture sets on the bulk permeability has been investigated during a hydraulic injection. It has been shown that their orientation for a given in-situ stress field plays a major role. However, if homogeneous properties are assigned to the fracture planes in the model, the limited irreversible displacements cannot be reproduced. Despite these limited displacements $(40 \mu \mathrm{m}$ maximum), the transmissivity increased by a factor of 10-100. These results provide insights in fracture controlled permeability of fault zones depending on the geometrical properties of the fractures and their resulting hydro-mechanical behavior for a given in-situ stress field.
\end{abstract}

\section{Introduction}

Recent field studies suggest that taking into account subcritical mechanical response may be required to explain why the permeability of a fault zone in shales can increase by a factor of 10-100 (reaching the milli-Darcy range) when increasing the fluid pressure during injection tests, while strain records do exhibit generally very low plastic deformation [1],[2]. This has been observed during fluid injection tests performed inside the clay-rich Tournemire fault zone, at the $250 \mathrm{~m}$ deep Tournemire Underground Research Laboratory (URL), Southern France. These injection tests were part of the "Fluids and Faults" project which was undertaken to constrain and better understand the relationship relating permeability, pressure, stress and strain in fault zones in shale for application to basin and reservoir modeling [3]. Based on the results obtained from this highly monitored project, we have set up a numerical model to investigate the hydromechanical conditions in which

\footnotetext{
* Corresponding author: frederic.donze@ univ-grenoble-alpes.fr
} 
the flow rate and permeability increase dramatically depending on the fluid pressure while the mechanical response of the hydraulically loaded zones, exhibits a relatively minor irreversible strain response.

\section{Tournemire in-situ experimental tests}

The in-situ field fluid injection experiments were performed at the Tournemire underground research laboratory (URL, Fig. 1a, top-right). The argillaceous formation where the URL is nested is $250 \mathrm{~m}$ thick and corresponds to sub-horizontal consolidated argillaceous and marly layer of lower Jurassic period [4]. The mineralogical composition of the Tournemire shale consists of 40 to $50 \%$ of phyllosilicates mainly smectite, illite, kaolinite, chlorite and mica, 10 to $20 \%$ of quartz, 10 to $30 \%$ of calcite and 4 to $8 \%$ of feldspars [5]. The shale is characterized by a low porosity, from 8 to $12 \%$, corresponding to a water content of 3.5 to $5 \%$ weight at saturation and a hydraulic conductivity of $10^{-14}$ to $10^{-15} \mathrm{~m} / \mathrm{s}\left(10^{-21}\right.$ to $\left.10^{-22} \mathrm{~m}^{2}\right)$ measured on core samples in the laboratory [6].

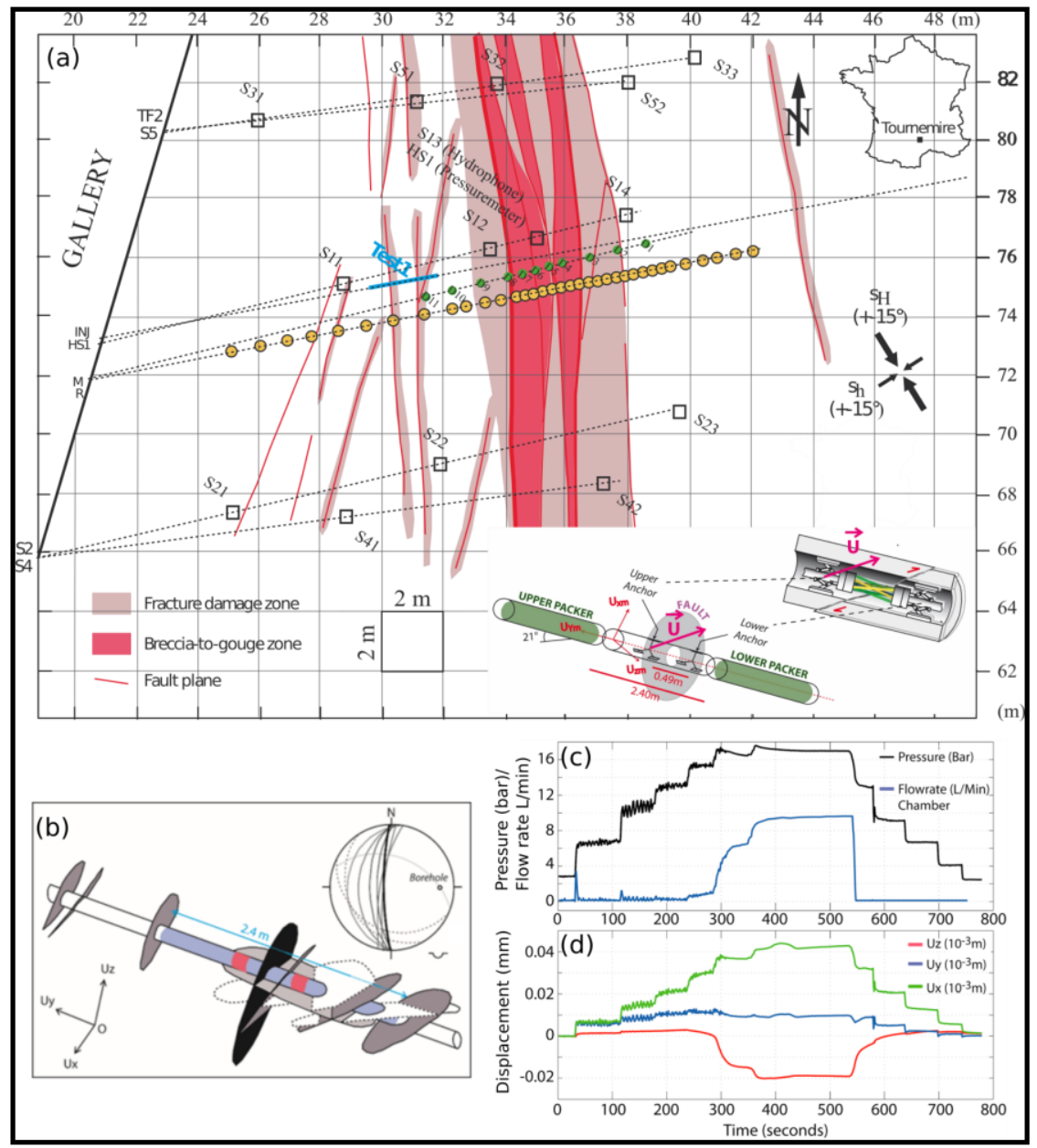

Fig 1. (a) Map view of the investigation area: Test 1 interval is shown in blue colour along the injection borehole, the monitoring probe is shown at the right bottom corner. (b) Test 1 interval geology where the secondary fault is shown in black. (c) Temporal evolution of the pressure and flow rate (upper graph) and (d) displacements (lower graph) -versus-time. 
The apparatus used for the fluid pressurization was composed of a probe (Fig. 1a, bottom-right) used to monitor 3D displacement between two points anchored to the borehole walls (Fig. 1c) at the same time as fluid pressure and flow rate (Fig. 1d). During test 1 (Fig. 1a), the water was injected at a low pressure for a given duration. The injection pressure was incremented and held constant, typically for the same duration (Fig. 1c). This was repeated while monitoring the deformations across the fault with the $3 \mathrm{D}$ deformation probe. After reaching a threshold pressure, a large increase in flow rate at constant pressure could be observed (Fig. 1c).

The temporal evolution of the displacements, recorded by the mechanical probe inside the injection chamber (Ux, Uy, Uz), are presented in Fig. 1d. At 237 seconds and for a pressure equal to $1.5 \mathrm{MPa}$, a large non-linear flow rate increase was observed that later reached a quasi-constant value (450-537 seconds) before the pressure was gradually decreased (Fig. 1c). Before 1.5 MPa there was no flow rate measured and at the onset of pressure decrease, the flow rate falls abruptly to zero without any further flow injected in the fault zone for the rest of the injection test. The mechanical response of the test showed that the induced displacements are not only characterized by a shear component much larger than the normal component (Fig. 1d) but they also it exhibits reversibility. The movement inside the fault zone was associated to a left-lateral slip which is in agreement with the measured direction of the principal stresses.

Several other series of tests were carried out and in all of them the transmissivitypressure dependency exhibited a substantial increase (more than $100 \mathrm{~mL} / \mathrm{min}$ ) only above a pressure threshold which has been called the Fault Opening Pressure (FOP) [3].

\section{Numerical modeling}

To reproduce this fluid propagation process from a geo-hydromechanical point of view, a discrete element model has been set up using the three-dimensional software 3DEC [7]. Besides the model containing the totality of the fractures identified crossing the injection chamber (Fig. 2a, case a), the contribution of each fracture family's orientation is also tested. More specifically, the secondary fault plane (i.e. the brown plane Fig. 2a, cases a, b and c) is modeled together with the sub-vertical fracture family only in a second configuration (Fig. 2b, case b) and in a third configuration, the fault is modeled with the sub-horizontal fracture family only (Fig. 2c, case c).

In all cases, the fracture plane that was mostly affected by the fluid pressurization is the secondary fault, i.e. the brown plane (Fig. 2, bottom). The rest of the fractures appear to be locally pressurized only at the injection point (point of intersection with the borehole) or at the intersection points with the pressurized fault but without any propagation taking place within their planes. So far, as the properties of the fractures considered are identical, the effect of the orientation with respect to the stress field is the controlling parameter. Keeping in mind that $\sigma_{\min }=\sigma_{\mathrm{y}}$ which is almost normal to the secondary fault, the fractures characterized by a dip angle lower than $40^{\circ}$ are not activated even for identical hydromechanical properties and with more favorable orientation for shear reactivation in the $z-y$ plane shown. 


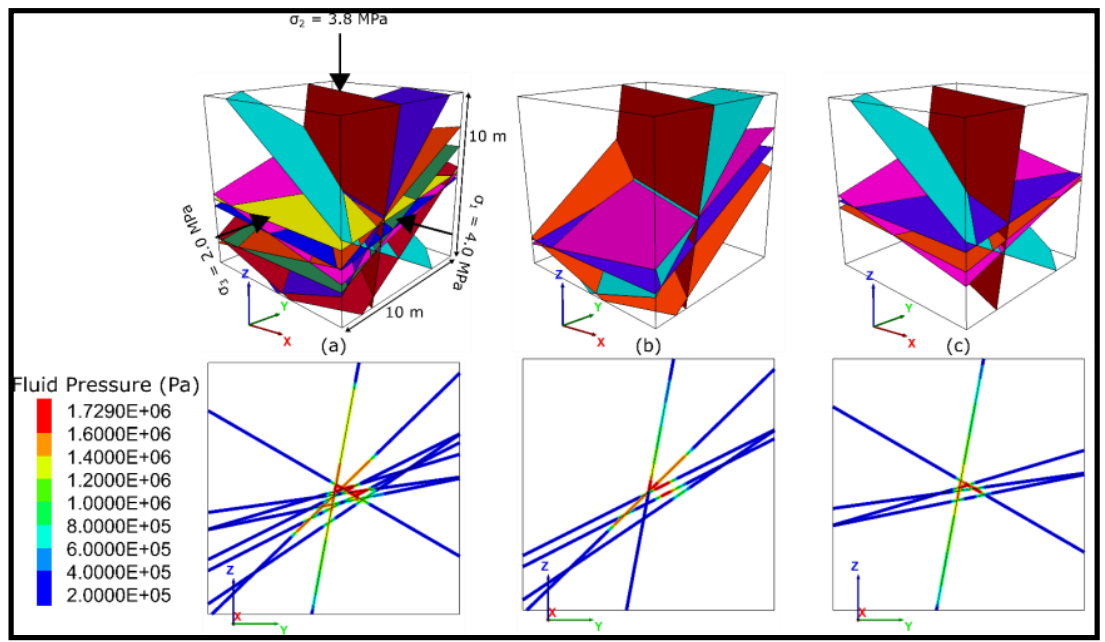

Fig. 2. Model geometry (top) and fluid pressure distribution shown along a vertical plane at the centre of the model (bottom) for $\mathrm{t}=537$ seconds corresponding to the end of the highest pressure test, for (a) the secondary fault plane with the sub-vertical and sub-horizontal families, (b) secondary fault plane with the sub-vertical family, (c) secondary fault plane with the sub-horizontal family.

The temporal evolution of the displacements calculated for these three configurations within the fluid injection zone, were then compared to the experimental data. Only the results, corresponding to the secondary fault plane with the sub-vertical family (case $b$ in Fig. 2) are represented in Figure 3. The displacement in the three directions (x,y and z) are quite well reproduced during the first 550 seconds (Fig. 3 left). However, the model was not able to reproduce correctly the reversible displacement observed once the fluid injection has been stopped.

In terms of flow rate response, reminding that the injection fluid pressure is imposed, an abrupt increase is observed (Fig. 3 right) for time $t=200$ seconds. This one is higher than the one observed in the experimental data set, because the vertical displacement, i.e. the zdirection (purple line) increases earlier in the model. This strong non-linearity response observed in the experiments is not well reproduced by the model. Finally, the constant flow rate value obtained in the models is closed to the one observed experimentally, until the unloading takes place (i.e. end of the fluid injection sequence).

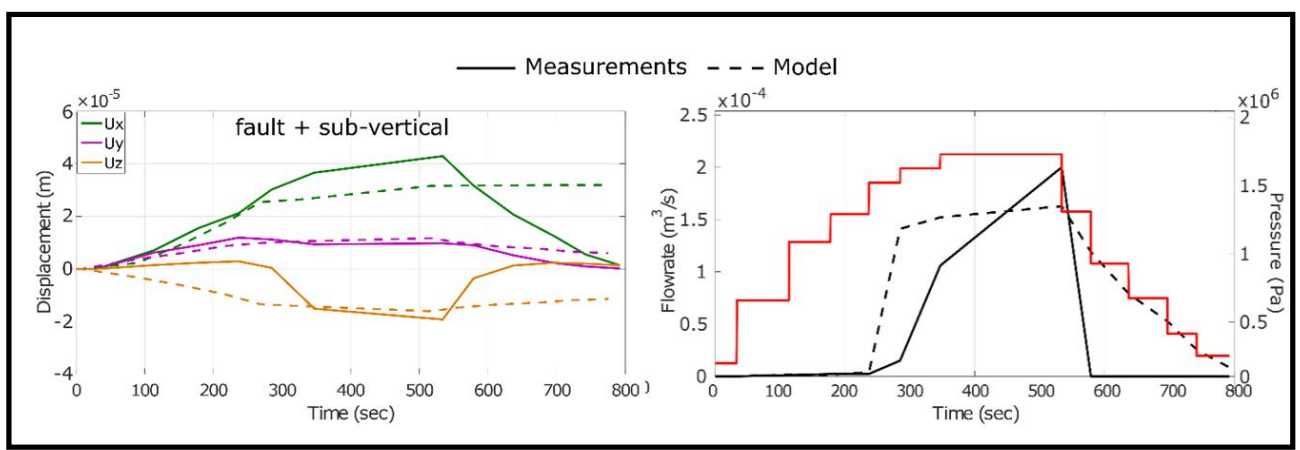

Fig. 3. Displacements and flow rate versus time corresponding to the secondary fault plane with the sub-vertical family only (case b on Fig. 2). On the left, the temporal evolution of the displacements for the three components is plotted, and on the right the temporal evolution of the flow rate and the applied fluid pressure in red. Dashed lines correspond to the numerical model results and the solid lines for the experimental data sets. 
Thus, models assuming homogeneous slipping plane could well reproduce data during the first steps of the pressurization tests associated to the hydraulic opening of the Tournermire tests (Fig. 1c), e.g. up to $550 \mathrm{~s}$ for Test 1, but they remain limited to explain the observed FOP associated to a reversible response in terms of displacement (Fig. 1d). The FOP concept associated to this limited mechanical response might be a consequence of the heterogeneous nature of the solicited rock volume. Taking into account the in-plane heterogeneities could explain the observed FOP with its increase of permeability and its limited slip motion while explaining explicitly the channelling process of the fluid.

\section{Conclusion}

The model confirmed that considering reasonable property values, very small displacements, i.e. at the dozen micrometers scale, along pre-existing discontinuities can dramatically increase the flow rate inside the rock mass at the decameter scale. The significant role of both, stress and orientation was quantified showing that for a low differential stress value, fractures forming an angle higher than $40^{\circ}$ with the maximum stress (dip angle lower than $50^{\circ}$ ) cannot be reactivated. The model shows that the relative orientation of the discontinuities within the stress field is of first order in the hydromechanical behavior of connected fractures.

Because field observations suggest the existence of flow paths in fault zones that transmit fluids rapidly over large distances, it seems that considering homogeneous hydromechanical properties along fractures as done in the present model, does not represent a reasonable hypothesis. Channeling process should be considered at the fracture scale in order to reproduce the dramatic increase of permeability increase above FOP, while ensuring a reversible deformation as the fluid pressure drops down.

Acknowledgements. The second author would like to thank Total for funding this research project (contract FR00006163).

\section{References}

1. Y. Guglielmi, et al., J Geophys Res: Solid Earth, 120, 7729-7748 (2015)

2. Y. Guglielmi, et al., Energy Proc, 114, 3167-3174 (2017).

3. P. Henry, et al., AGU Fall Meeting Abstracts, \#T54B-04 (2016)

4. J.M. Matray, et al., Eng Geolo, 90, 1-16 (2007)

5. J. Tremosa, et al., Appl Geochem, 27, 1417-1431 (2012)

6. J.Y. Boisson, L. Bertrand, J. F. Heitz, \& Y. Golvan,. Hydrogeol Jour, 9, 108-123 (2001)

7. Itasca Consulting Group Inc. (Itasca) 3DEC (3-Dimensinal Distinct Element Code), version 5.2, Itasca, Minneapolis (2015) 